\title{
Maggot therapy in treatment of a complex hand injury complicated by mycotic infection
}

\author{
Bohac $\mathrm{M}^{1}$, Cambal $\mathrm{M}^{2,4}$, Zamborsky $\mathrm{R}^{5}$, Takac $\mathrm{P}^{3,4}$, Fedeles $\mathrm{J} \mathrm{Sr}^{1}$ \\ Department of Plastic Surgery, University Hospital Bratislava, Faculty of Medicine Comenius University \\ in Bratislava, Bratislava, Slovakia. bohac.md@gmail.com
}

\begin{abstract}
Complex injuries of the hand remain a therapeutic challenge for surgeons. We present the case of a male who suffered a devastating injury of the hand caused by a conveyor belt. The patient developed a progressive $A b$ sidia corymbifera infection of the affected soft tissues. Initial treatments with serial surgical debridement and topical and intravenous itraconazole were unsuccessful in eliminating the infection. We decided to use maggot debridement therapy in a new special design to debride all necrotic, devitalized tissue and preserve only healthy tissue and functioning structures. This maneuverer followed by negative pressure therapy allowed progressive healing. In such complex hand injuries, maggot debridement combined with negative pressure therapy could be considered to achieve effective and considerable results, although future functional morbidity may occur (Fig. 4, Ref. 18). Text in PDF www.elis.sk.

KEY WORDS: maggot debridement therapy, negative pressure therapy, complex hand injuries, mycotic infection.
\end{abstract}

\section{Introduction}

Complex hand injuries are multi-structural (soft tissue, bones, vessels, tendons, and nerves) injuries with loss of function and sensitivity or loss of the affected body part (1).

Pathogenic microorganisms are frequent complicating factors of such severe injuries. However, mycotic infection puts patients at a particularly increased risk. Invasive mycotic diseases are common in immunocompromised patients and individuals with other significant comorbidities (5-7). However, several infections of the soft tissue by Absidia corymbifera (syn. Lichtheimia corymbifera, Mycocladus corymbifer) have been reported in patients without immune dysfunction who had experienced traumatic injuries contaminated with soil (2-4). A. corymbifera is the only member of

\footnotetext{
${ }^{1}$ Department of Plastic Surgery, University Hospital Bratislava, Faculty of Medicine Comenius University in Bratislava, Bratislava, Slovakia, ${ }^{2} 1 \mathrm{st}$ Department of Surgery, University Hospital Bratislava, Faculty of Medicine Comenius University in Bratislava, Bratislava, Slovakia, ${ }^{3}$ Institute of Zoology, Molecular and Applied Zoology, Slovak Academy of Sciences, Bratislava, Slovakia, ${ }^{4}$ Scientica, sro, Bratislava, Slovakia, and ${ }^{5}$ Department of Traumatology, Faculty of Medicine, Slovak Medical University, Bratislava, Slovakia
}

Address for correspondence: M. Bohac, MD, PhD, Department of Plastic Surgery, University Hospital Bratislava, Medical Faculty of Comenius University in Bratislava, Ruzinovska 6, SK-826 06 Bratislava, Slovakia. Phone: +421.903316429,

Acknowledgements: This work was funded by the Operational Program 'Research and Development' and co-financed by the European Fund for Regional Development (EFRD) via grant ITMS 26240220030 - "Research and development of new biotherapeutic methods and their application in the treatment of some illnesses".

The VivanoTec ${ }^{\mathrm{R}}$ device is manufactured by Hartmann Inc. (DE). the Zygomycetes phylum recognized to be a human pathogen $(14,15)$. Treatment efforts for this infection must prevent local and systemic spread because mycotic dissemination is associated with high mortality and amputation rates (8-10). Removal of all necrotic tissue and microbiological diagnosis with target antimicrobial therapy are crucial steps in its early management.

There are a number of existing methods for debridement including mechanical, surgical, autolytic, and enzymatic methods. Each of these techniques has associated disadvantages, such as limited efficacy, need for anesthesia, pain, and mechanical damage to the underlying healthy tissue. Maggot debridement therapy (MDT) is a technique that has recently attracted some attention (11). The proven behaviors of MDT are considerable debridement, granulation support, and antimicrobial effects (12).

Negative pressure therapy (NPT) is useful after thorough debridement to promote wound healing by increasing local blood flow and open wound closure (13).

To the best of our knowledge, this is the first reported case of tendon and soft tissue infection by A. corymbifera in a complex hand injury that was successfully treated with a combination of itraconazole, MDT and NPT.

\section{Patient}

A previously healthy 21-year-old male had a work accident at a conveyor belt, which caused a devastating injury to his upper left extremity. He was transferred to our department from the district trauma hospital, where he had undergone fasciotomies and surgical debridement two days after the accident. After the patient arrived at our hospital, the affected extremity was evaluated. Limited vitality of the soft tissue and extensor tendon over the width of the dorsal 


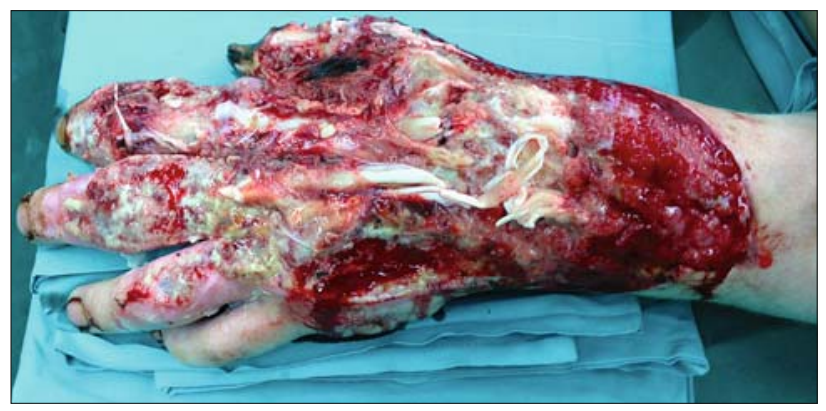

Fig. 1. The patients' first photo of the hand before debridement at our department.

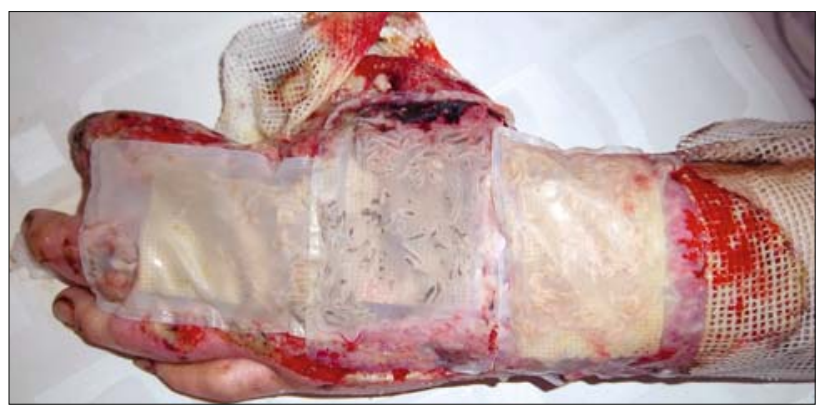

Fig. 2. The first application of maggot debridement therapy. In the central bag, full (black) maggots can be observed because of the large amount of necrosis.

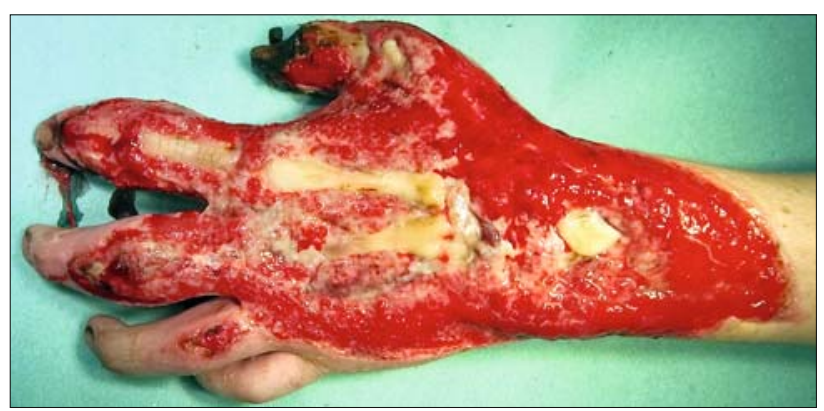

Fig. 3. The patients' hand immediately after two applications of maggot debridement therapy.

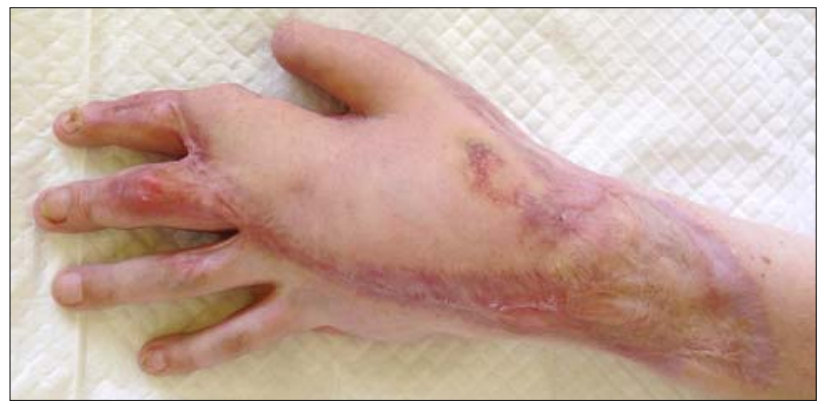

Fig. 4. The patients' hand after groin flap and full-thickness skin graft coverage.

distal third of the forearm and dorsum of the hand to nearly the end of the first three fingers was observed (Fig. 1). After the initial surgical debridement, antibiotic treatment with vancomycin, gentamicin, and metronidazole was started. The first wound cultures showed colonization by Staphylococcus haemolyticus. During several debridement procedures, we attempted to remove all necrotic tissue, but the pathological process slowly progressed. Six days after our first intervention, wound cultures revealed colonization by A. corymbifera. According to the pharmacist recommendation, we started antimycotic therapy with itraconazole. At this time, a new treatment approach was attempted for precise debridement. We decided to use MDT in a new special design, using the eggs of $L$. sericata, in contrast to the more common use of maggots. The experimental protocol was approved by the Ethical committee of the University Hospital and Faculty of Medicine, Comenius University (Reference number 112/2005). The patient gave informed consent before the beginning of MDT. Bags with sterile eggs of $L$. sericata were obtained from Scientica, Ltd. (St. Michael's Hospital, Bratislava, Slovakia) and were prepared individually for patient to match the size of the wound. The biobags were placed onto the wound. On the top, saline-moistened gauze was placed and secured well with secondary dressing to avoid slippage and ensure that surface contact of the biobag is maintained. All outer dressing must be non-occlusive as the larvae need oxygen to survive. The secondary dressing was changed daily. The bags were applied for approximately 72 hours, depending on the patient's tolerance. After two applications of three egg biobags (maggots were delivered from the eggs) (Fig. 2), we achieved a significant improvement in the wound condition (Fig. 3). The maggots removed all necrotic tissues, and the mycotic infection was successfully eradicated. However, the wound showed massive colonization by Proteus mirabilis, which is a typical commensal organism in the $L$. sericata maggot's gut and was sensitive to all common antibiotics. Additionally, early stages of granulation and exposure of the carpal, metacarpal, phalangeal bones and ends of the extensor tendons were observed. The wound was not suitable for complex flap reconstruction in this condition. Ciprofloxacin and NPT (VivanoTec ${ }^{\mathrm{R}}$ Hartmann) were used to promote granulation and treat the infection before definitive wound covering. NPT was activated on $125 \mathrm{mmHg}$ intermittent suction pressure. Dressings were changed in the isolation ward once every 3 to 4 days under strict aseptic conditions. After five weeks of NPT, granulation tissue covered all exposed tendons and only the rest of exposed bones remained. The swelling decreased, and the wound contracted, leaving a total defect in size of $300 \mathrm{~cm}^{2}$. According to angiography findings of the a. radialis occlusion, we chose to cover the defect with a pedicle groin flap of 12 x $18 \mathrm{~cm}$ instead of a free flap. The rest of the surrounding defect was covered with a split-thickness skin graft. Three weeks after the last operation had been performed, the flap was disconnected, and the patient was discharged (Fig. 4). During six months of follow-up, no infection relapses occurred, and the patient is currently undergoing rehabilitation and waiting for flap defatting and extensor tendon reconstruction.

\section{Discussion}

There is a lack of information in literature to guide the treatment of such complex hand injuries complicated by mycosis. The few series reporting soft tissue zygomycosis suggest that 
the highest survival rates are obtained using a combination of extensive debridement and long-term antifungal treatment (16). The overall mortality of cutaneous $A$. corymbifera infection was $18.5 \%$, although in neonates, it can reach more than $50 \%$ (8). The typical clinical feature of cutaneous zygomycosis is tissue necrosis with a rapid clinical course. Early diagnosis and target treatment are essential for a good outcome. Although some in vitro studies of Zygomycetes have demonstrated that the Absidia genus of fungi is sensitive to amphotericin $B$ and relatively low concentrations of itraconazole and terbinafine, further studies correlating in vitro and in vivo findings are needed (17). In cases involving the hand structures, debridement should be adequate in regard to future function, and the advantages of MDT are useful. The literature and our own results demonstrate that MDT is a safe method with few side effects. MDT is as good as or better than conventional surgical debridement, is more selective than surgical debridement, and decreases the time to healing and duration of stay in the ward (18). NPT has also been shown to have good results in the management of open chronic wounds. This is the first reported case of a complex hand injury complicated by zygomycosis and treated with a combination of antifungal therapy, MDT and NPT.

\section{References}

1. Eisenschenk A, Ekkernkamp A, Stengel D, Zach A, Farwick JH. A new pathway to classification of complex hand injuries: a clinical pilot testing using a new method of assessment. Arch Orthop Trauma Surg 2012; 132 (9): 1343-1351.

2. Spellberg B, Edwards JJ, Ibrahim A. Novel perspectives on mucormycosis: pathophysiology, presentation, and management. Clin Microbiol Rev 2005; 18 (3): 556-569.

3. Gordon G, Indeck M, Bross J, Kapoor DA, Brotman S. Injury from silage wagon accident complicated by mucormycosis. J Trauma 1988; 28: 866-867.

4. Cocanour CS, Miller-Crotchett P, Lawrence Reed R, Johnson PC, Fischer RP. Mucormycosis in trauma patients. J Trauma 1992; 32: 12-15.

5. Ingram CW, Sennesh J. Disseminated zygomycosis: report of four cases and review. Rev Infect Dis 1989; 11: 741-754.
6. Nagy-Agren SE, Chu P, Walter Smith GJ, Waskin HA, Altice FL. Zygomycosis (mucormycosis) and HIV infection: report of three cases and review. J Acquired Immun Defic Syndrom Hum Retrovirolo1995; 10: 441-449.

7. Levy E, Bia MJ. Isolated renal mucormycosis: case report and review. J Amer Soc Nephrol 1995; 15: 2014-2019.

8. Almaslamani M, Taj-Aldeen S, Garcia-Hermoso D et al. An increasing trend of cutaneous zygomycosis caused by Mycocladus corymbifer (formerly Absidia corymbifera): report of two cases and review of primary cutaneous Mycocladus infections. Med Mycol 2009; 47 (5): 532-538.

9. Chamilos G, Lewis R, Kontoyiannis D. Delaying amphotericin Bbased frontline therapy significantly increases mortality among patients with hematologic malignancy who have zygomycosis. Clin Infect Dis 2008; 47 (4): 503-509.

10. Spellberg B, Walsh T, Kontoyiannis D et al. Recent advances in the management of mucormycosis: from bench to bedside. Clin Infect Dis 2009; 48 (12): 1743-1751.

11. Zarchi K, Jemec GBE. The efficacy of maggot debridement therapy - a review of comparative clinical trials. Int Wound J 2012. doi: 10.1111/j.1742-481X.2011.00919.x

12. Cambal M et al. Maggot therapy and its use in clinical praxis. 2012, Medical scripts.

13. Caniano D, Ruth B, Teich S. Wound management with vacuum- assisted closure: experience in 51 pediatric patients. J Pediatr Surg 2005; 40 (1): 128-132.

14. Ribes JA, Vanover-Sams CA, Baker DJ. Zygomycetes in hu- man disease. Clin Microbiol Rev 2000; 13: 236-301.

15. Cloughley R, Kelehan J, Corbett-Feeney G et al. Soft tissue infection with Absidia corymbifera in a patient with idiopathic aplastic anemia. J Clin Microbiol 2002; 40: 725-727.

16. Zaoutis T, Roilides E, Chiou $\mathbf{C}$ et al. Zygomycosis in children: a systematic review and analysis of reported cases. Pediatr Infect Dis J 2007; 26 (8): 723-727.

17. Dannaoui E, Meletiadis J, Mouton JW et al. In vitro suscep- tibilities of zygomycetes to conventional and new antifungals. J Antimicrob Chemother 2003; 51: 45-52.

18. Gottrup F, Jorgensen B. Maggot Debridement: An Alternative Method for Debridement. WWw.eplasty.com, 2011, 11: 290-302.

Received February 5, 2015. Accepted June 26, 2015. 